\title{
3D-SIMULATION STUDIES OF SNS RING DOUBLET MAGNETS*
}

\author{
J. G. Wang", SNS/ORNL, Oak Ridge, TN 37831-6471, U.S.A. \\ N. Tsoupas, BNL, Upton, NY 11973, U.S.A. \\ M. Venturini, LBNL, Berkeley, CA 94720, U.S.A.
}

\begin{abstract}
The accumulator ring of the Spallation Neutron Source (SNS) at ORNL employs in its straight sections closely packed quadrupole doublet magnets with large aperture of $\mathrm{R}=15.1 \mathrm{~cm}$ and relatively short iron-to-iron distance of $51.4 \mathrm{~cm}$. These quads have much extended fringe field, and magnetic interferences among them in the doublet assemblies is not avoidable. Though each magnet in the assemblies has been individually mapped to high accuracy of lower $10^{-4}$ level, the experimental data including the magnet interference effect in the assemblies will not be available. We have performed 3D computing simulations on a quadrupole doublet model in order to assess the degree of the interference and to obtain relevant data for the SNS ring commissioning and operation.
\end{abstract}

\section{INTRODUCTION}

The SNS accumulator ring contains four straight sections, each of which employs two quadrupole doublets (as shown in Fig. 1) for beam focusing. The doublet quadrupoles are designated as 30Q58 and 30Q44, where 30 is the magnet aperture in $\mathrm{cm}$, and 58 and 44 are the magnet steel length in $\mathrm{cm}$ for the two quads respectively. The iron-to-iron distance between the two quads is $\mathbf{5 1 . 4}$ $\mathrm{cm}$. In addition, there is a dipole corrector 41CD30 in the assembly, whose iron core is $21.4 \mathrm{~cm}$ away from the iron core of 30Q58. The small aspect ratio, defined as the steel length over the aperture diameter of the quads, leads to significant fringe fields. The relatively smaller iron-toiron distances cause concerns about the magnetic interferences between the two quads, as well as between 30Q58 and 41CD30.

The ring doublet magnets were designed at BNL $[1,2]$ with the simulation tool of both 2D and 3D OPERA/TOSCA [3]. The magnets were built in Russia and measured individually with high accuracy at BNL. But, there is no magnetic measurement of the doublet assembly, and thus the magnetic interferences among the three magnets are unknown. This has motivated us to study the issue through 3D computing simulations.

\section{SIMULATION PROCEDURES}

The simulation code we use is also OPERA/TOSCA with its new version 10.01. The simulation models are built with the OPERA3d package "Modeller", which makes easier to simulate two or more magnets together. The magnet dimensions in the models follow exactly the BNL design drawings [4].

*SNS is managed by UT-Battelle, LLC, under contract DE-AC0500OR22725 for the U.S. Department of Energy.

\#igwang@ornl.gov

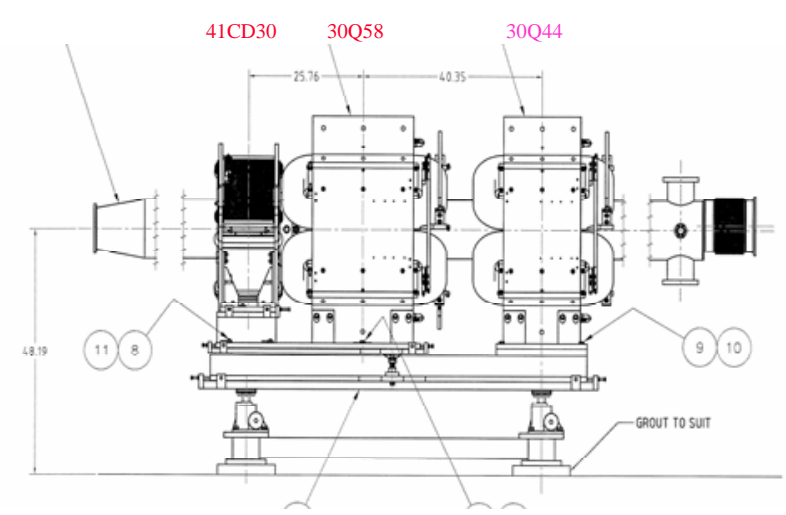

Figure 1: SNS ring doublet assembly [4].

We have first simulated 30Q58 and 30Q44 separately. In the simulation the currents are $810 \mathrm{~A}$ for $30 \mathrm{Q} 58$ and -860 A for 30Q44, respectively, which are close to their operation points for $1 \mathrm{GeV}$ beam energy. We then combine the two magnets together in one simulation. The output will provide the magnetic interference between 30Q58 and 30Q44. Finally, we add the iron core of $41 \mathrm{CD} 30$ to the assembly for simulation. Figure 2 shows the simulation model of 30Q58, while in Fig. 3 we plot the magnetic field component $B_{y}$ versus $z$ at $x=10 \mathrm{~cm}$, $\mathrm{y}=0$. Similar figures are also produced for 30Q44.
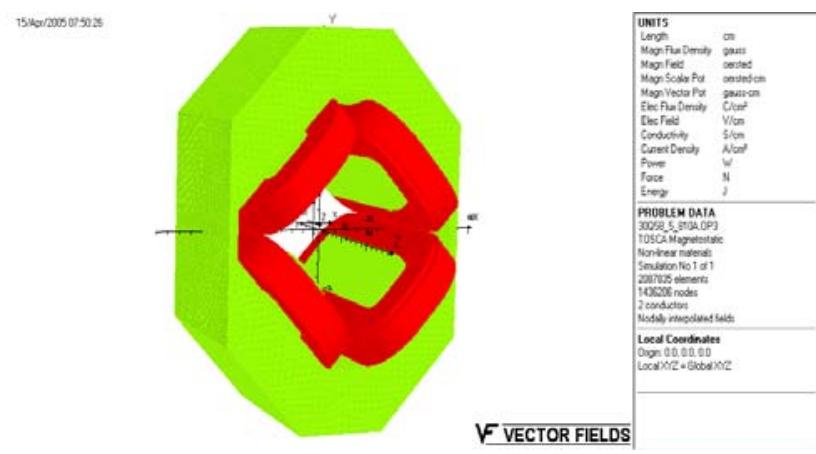

Figure 2: Simulation model of 30Q58.

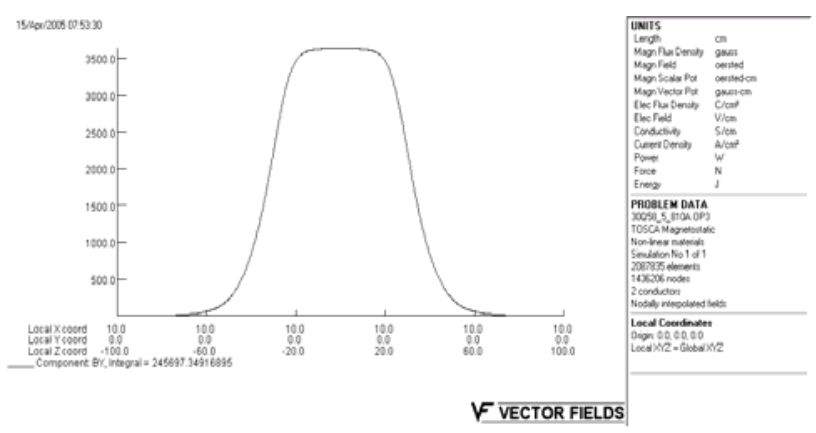

Figure 3: $B_{\mathrm{y}}$ vs. $\mathrm{z}$ at $\mathrm{x}=10 \mathrm{~cm}, \mathrm{y}=0$. 
In post-processor calculations the integrated gradients and harmonic contents are obtained by Fourier-analyzing the magnetic flux through a rectangular patch rotating about the z-axis. The patch has a dimension of $r_{4}=14.224$ $\mathrm{cm}$ and $\mathrm{r}_{2}=-7.112 \mathrm{~cm}$ with various lengths, which is similar to the un-bucked winding of a Halbach coil in measurements [5]. The integrated gradient of 30Q58 alone is $2.4597 \mathrm{~T}$, and the dodecapole and $20^{\text {th }}$ pole are 4.0 and 7.4 units, respectively. For 30Q44 alone, the integrated gradient is $2.0721 \mathrm{~T}$, and the dodecapole and $20^{\text {th }}$ pole are 4.4 and 7.9 units, respectively. The harmonics are evaluated at a radius of $10 \mathrm{~cm}$.

The z-dependent parameters of the magnets are obtained by 3D multipole expansions [6, 7]. The magnetic field component $B_{r}$ on a cylindrical surface of $\mathrm{R}=10 \mathrm{~cm}$ is calculated and Fourier-decomposed. The generalized gradients, which constitute the magnetic field components in the 3D multipole expansion, can then be constructed. Figures 4 \& 5 show b2 and b6 terms for 30Q58 alone, which correspond to the linear gradient and dodecapole terms. Note that the plots in the two figures are only made for the positive $\mathrm{z}$ values. The reflection about $\mathrm{z}=0$ does apply for a single quad due to the symmetry about $\mathrm{z}=0$. Similar plots are also made for 30Q44 alone and for the cases in the doublet assembly.

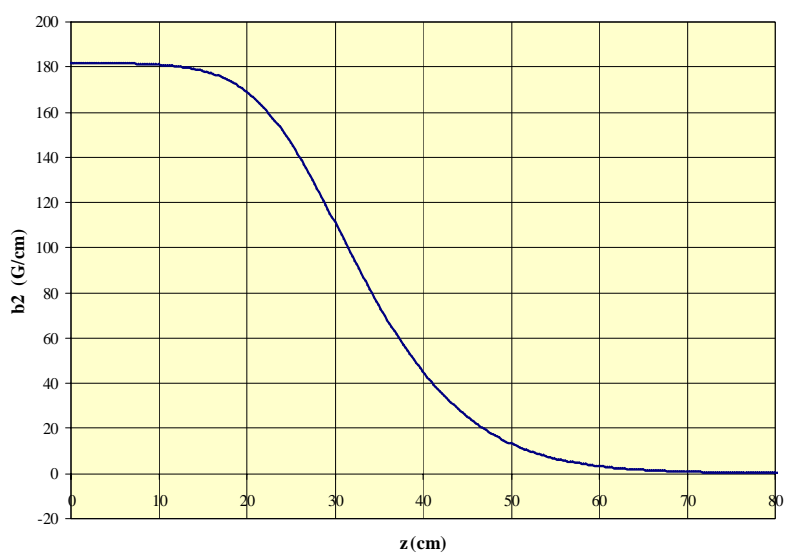

Figure 4: Linear gradient b2 vs. $\mathrm{z}$ for 30Q58.

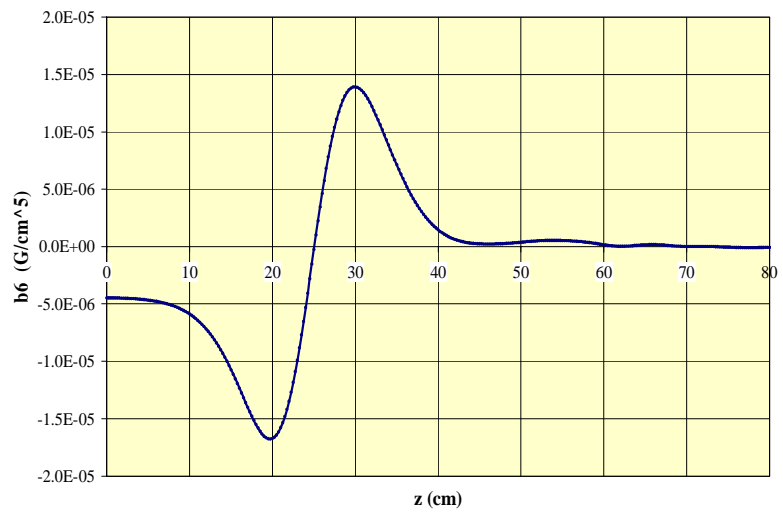

Figure 5: Generalized gradient b6 vs. z for 30Q58.
The simulations with the two quads or the two quads plus a corrector result in a rather large output file of about 1.6 GB in memory. We often encounter difficulties in volume mesh, and it is very slow to do this with even a powerful PC.

\section{MAGNETIC INTERFERENCE}

Figure 6 shows the simulation model of the two quads, where the magnet currents are the same as for each single magnet. In the setting the center-to-center distance between 30Q58 and 30Q44 is $102.489 \mathrm{~cm}$.

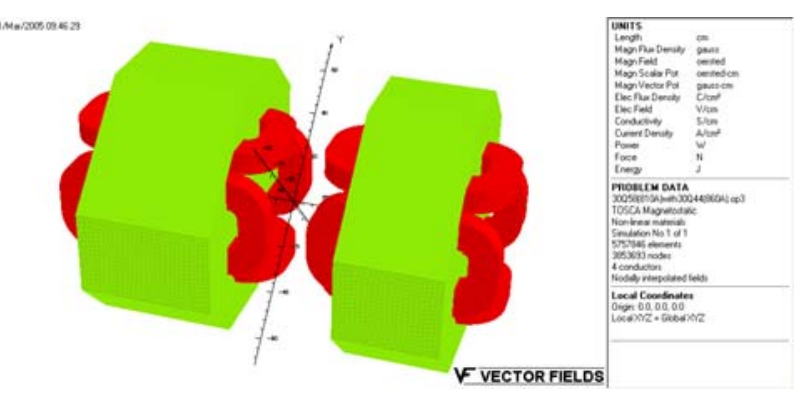

Figure 6: Simulation model for two doublet magnets.

In Fig. 7 we plot the magnetic field component $\mathrm{B}_{\mathrm{y}}$ at $\mathrm{x}=10 \mathrm{~cm}$ and $\mathrm{y}=0$ as a function of the axial position $\mathrm{z}$ for three cases: the dark blue curve represents the field of 30Q58 alone; the light blue curve shows the field of 30Q44 itself; the red curve is the field when the two quads are together in simulation. It is apparent that the integrated field, as well as the integrated gradient, gets smaller for each magnet in the doublet.

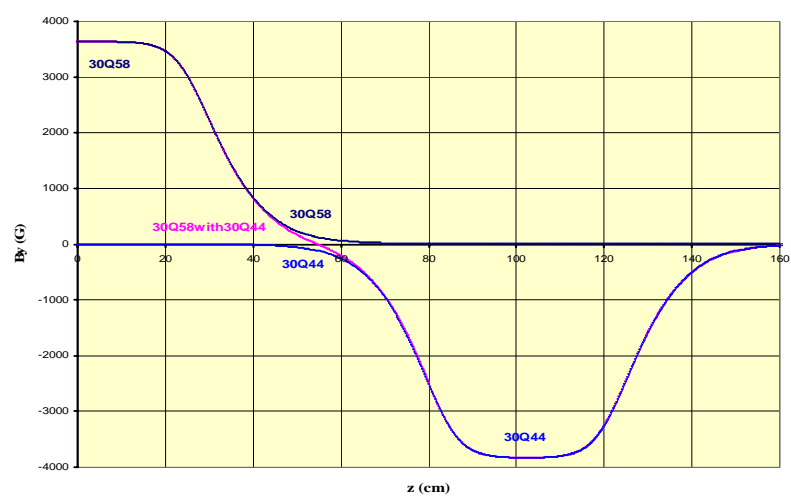

Figure 7: By vs. $\mathrm{z}$ at $\mathrm{x}=10 \mathrm{~cm}$ and $\mathrm{y}=0$ for the two quads.

The quantitative reduction in the integrated gradients due to the interference is calculated in the same way as for the single magnet. In the case of two quads, the above field component $\mathrm{B}_{\mathrm{y}}$ crosses zero at $\mathrm{z}=54.51 \mathrm{~cm}$ between the two quads. This point is used naturally as the separation point of the two quads during the calculation. The integrated gradient is $2.4434 \mathrm{~T}$ for $30 \mathrm{Q} 58$ and 2.0555 $\mathrm{T}$ for $30 \mathrm{Q} 44$ with a $-90^{\circ}$ phase difference. In comparison with the single magnet, the integrated gradients of the two quads are reduced by $0.66 \%$ and $0.80 \%$, respectively.

The final configuration of the simulation model is to add the corrector to the doublet, as shown in Fig. 8. Here, the center-to-center distance between 30Q58 and 41CD30 
is $65.4304 \mathrm{~cm}$. The corrector in the model has its iron core only. The magnetic field component $B_{y}$ at $x=10 \mathrm{~cm}$ and $\mathrm{y}=0$ as a function of the axial position $\mathrm{z}$ is plotted in Fig. 9 for three different cases as before. The field component $\mathrm{B}_{\mathrm{y}}$ crosses zero at $\mathrm{z}=54.66 \mathrm{~cm}$ between the two quads. The calculated integrated gradients are 2.4311 $\mathrm{T}$ for 30Q58 and 2.0550 $\mathrm{T}$ for 30Q44. The reduction in the integrated gradients is $1.16 \%$ for 30Q58 and $0.83 \%$ for 30Q44. In Table 1 we list these results together, where "In Doublet" means two quads only, while "In Assembly" refers to all three magnets together, i.e. two quads plus a corrector.

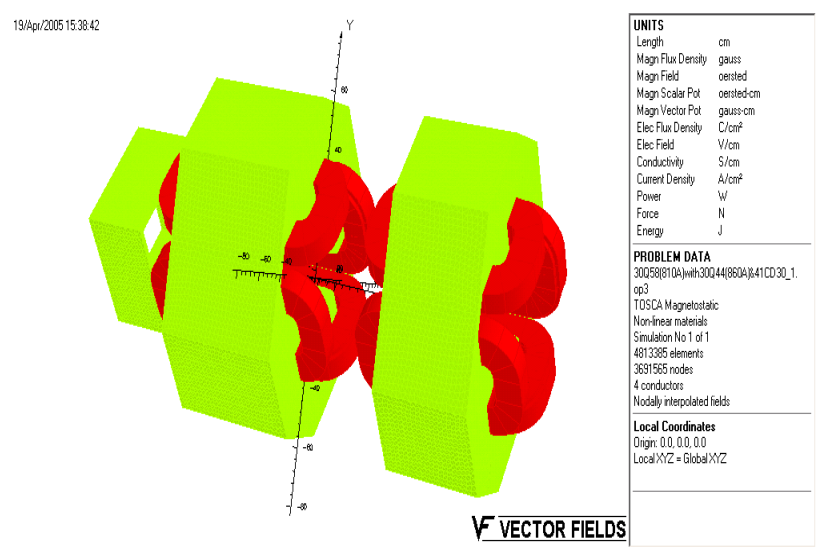

Figure 8: Model for two quads plus a corrector.

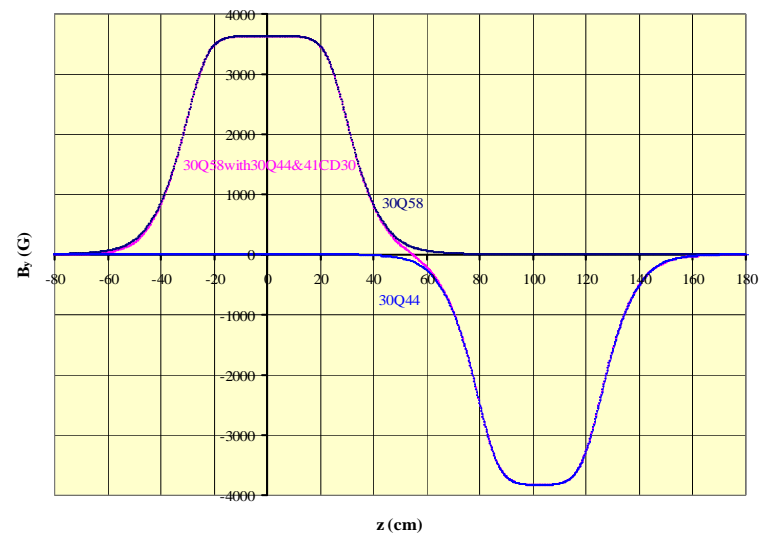

Figure 9: $\mathrm{B}_{\mathrm{y}}$ vs. $\mathrm{z}$ at $\mathrm{x}=10 \mathrm{~cm}$ and $\mathrm{y}=0$ for two quads plus a corrector.

Table 1 Changes in integrated gradients

\begin{tabular}{cll} 
& $\underline{30 Q 58}$ & $\underline{30 Q 44}$ \\
As Singlet: & $2.4597(\mathrm{~T})$ & $2.0721(\mathrm{~T})$ \\
In Doublet: & $2.4434(\mathrm{~T})$ & $2.0555(\mathrm{~T})$ \\
(Reduction): & $(0.66 \%)$ & $(0.80 \%)$ \\
In Assembly: & $2.4311(\mathrm{~T})$ & $2.0550(\mathrm{~T})$ \\
\multicolumn{1}{c}{ (Reduction): } & $(1.16 \%)$ & $(0.83 \%)$
\end{tabular}

The z-dependent parameters for the doublet and for the assembly are also obtained in the same way as for the singlet. These data will be used in future analyses.
In the simulation the harmonic contents of the two quads do not change appreciably from a singlet to doublets or in the assembly. These quantities are also very sensitive to mesh conditions. That makes it difficult to draw an accurate conclusion.

\section{SUMMARY}

3D computing simulations have been performed to study the performance characteristics of two narrow quads in the SNS ring doublet section. Especially, the magnetic interferences between the two quads and with a corrector have been investigated in modeling. The reduction of the integrated gradients due to the magnetic interferences can reach more than $1 \%$. This effect is very significant, considering the accuracy of the magnetic field requirement for the SNS ring is at lower $10^{-4}$ level.

A better description of magnetic fringe field and interferences is to compare the changes in transfer maps between a single quad and that in a doublet or the assembly. This can be done by employing magnetic field data from the 3D multipole expansions as mentioned above for accurate computation of transfer maps [6]. In fact, this is our on-going work and its result will be reported elsewhere in future.

We also plan to continue the simulation of the SNS ring doublet magnets at different current settings. This will provide not only new information for $1.3 \mathrm{GeV}$ operation, but also the dynamic nature of magnetic interferences.

\section{ACKNOWLEDGEMENTS}

The author would like to thank J. Simkin of Vector Field, England, for his help in many details of the OPERA code and simulation technique.

\section{REFERENCES}

[1] N. Tsoupas, et. al. "A Large-aperture Narrow Quadrupole for the SNS Accumulator Ring”, Proc. EPAC 2002, p.1106, Paris, June 3-7, 2002.

[2] N. Tsoupas et. al, "Magnetic Field Calculations for a Large Aperture Narrow Quadrupole", in Proc. PAC 2003, Portland, Oregon, May 12-16, 2003

[3] OPERA-3d (an OPerating environment for Electromagnetic Research and Analysis) is the pre and post processing system for electromagnetic analysis programs such as TOSCA (for non-linear magnetostatic or electrostatic field and current flow problems) developed by Vector Fields Limited, England.

[4] http://dwg-server.c-ad.bnl.gov/eng-arch/source.htm

[5] K. Halbach, "The Hilac Quadrupole Measurement Equipment”, Engineering Note, LBL, March 3, 1972.

[6] M. Venturini and A. Dragt, "Accurate Computation of Transfer Maps from Magnetic Field Data”, NIM (A) 427 (1999) 387-392.

[7] J. G. Wang, "Field Distributions of Injection Chicane Dipoles in SNS Ring”, in these Proceedings. 